\title{
Caracterização das residências multiprofissionais em saúde do Brasil
}

\author{
Characterization of multiprofessional healthcare residencies in Brazil
}

Caracterización de las residencias multiprofesionales de salud en Brasil

Mariana Barbosa da Silva ${ }^{1 *}$, Elizabeth Moura Soares de Souza², Patrícia Brazil Pereira Coelho ${ }^{3}$,
Paulo Sergio Gomes da Silva ${ }^{3}$, Camila Moreira Vasconcelos².

\section{RESUMO}

Objetivo: Caracterizar os programas de residências multiprofissionais em saúde do Brasil. Método: Estudo quantitativo, descritivo, transversal. Os dados foram coletados na internet por arquivos cedidos pelo Ministério da Educação e dos editais dos programas de residência, de março a setembro de 2019, através de um instrumento contendo as variáveis: região geográfica, estado brasileiro, financiamento, número de vagas, ano de início do programa, área de concentração, instituição propositora, profissões existentes e cenários de prática. Resultados: Os programas de residência estão concentrados na região sudeste, destacando-se 0 Estado de São Paulo. São Financiados, em sua maioria, pelo Ministério da Saúde, estando concentrados em instituições públicas. A profissão de enfermagem está inserida em grande parte dos programas. $O$ eixo de concentração na atenção básica/saúde é o mais ofertado. Conclusão: Os programas de residência multiprofissionais em saúde do Brasil são ofertados em todas as regiões do país, a maioria com bolsas do ministério da saúde, em instituições públicas, na área de concentração da atenção básica, sendo a enfermagem a profissão que está inserida em grande parte dos programas. A característica central das residências multiprofissionais em saúde é ser ensino em serviço, o que concretiza a formação interprofissional.

Palavras-Chave: Saúde, Internato não-médico, Especialização, Educação de pós-graduação, Capacitação profissional.

\begin{abstract}
Objective: To characterize the multiprofessional health residency programs in Brazil. Method: Quantitative, descriptive, cross-sectional study. The data were collected on the internet through files provided by the Ministry of Education and from the residency program notices, from March to September 2019, using an instrument containing the variables: geographic region, Brazilian state, financing, number of vacancies, year of beginning of the program, concentration area, proposing institution, existing professions and practice scenarios. Results: Residency programs are concentrated in the southeastern region, with the state of São Paulo standing out. They are mostly financed by the Ministry of Health, and are concentrated in public institutions. The nursing profession is part of most programs. The axis of concentration in primary care / health is the most offered. Conclusion: Multiprofessional health residency programs in Brazil are offered in all regions of the country, most with grants from the Ministry of Health, in public institutions, in the area of concentration of primary care, with nursing being the profession that is inserted in most programs. The central characteristic of multiprofessional residences in health is to be teaching in service, which concretizes the interprofessional training.
\end{abstract}

Keywords: Health, Non-medical internship, Specialization, Graduate education, Professional training.

\footnotetext{
1 Universidade Estadual de Ciências da Saúde de Alagoas (UNCISAL), Maceió - AL.

*E-mail: barbosamari@hotmail.com

2 Universidade Federal de Alagoas (UFAL), Maceió - AL.

3 Hospital Universitário Professor Alberto Antunes (HUPAA/UFAL), Maceió - AL.
} 


\section{RESUMEN}

Objetivo: Caracterizar los programas de residencia en salud multiprofesional en Brasil. Método: Estudio cuantitativo, descriptivo, transversal. Los datos fueron recolectados en Internet a través de archivos proporcionados por el Ministerio de Educación y de los avisos del programa de residencia, de marzo a septiembre de 2019, utilizando un instrumento que contiene las variables: región geográfica, estado brasileño, financiamiento, número de vacantes, año de inicio del programa, área de concentración, institución proponente, profesiones existentes y escenarios de práctica. Resultados: Los programas de residencia se concentran en la región sureste, destacando el estado de São Paulo. En su mayoría son financiados por el Ministerio de Salud y se concentran en instituciones públicas. La profesión de enfermería forma parte de la mayoría de los programas. El eje de concentración en atención primaria / salud es el más ofrecido. Conclusión: Los programas de residencia multiprofesional en salud en Brasil se ofrecen en todas las regiones del país, la mayoría con becas del Ministerio de Salud, en instituciones públicas, en el área de concentración de la atención primaria, siendo la enfermería la profesión que se inserta en la mayoría de los programas. La característica central de las residencias multiprofesionales en salud es la docencia en servicio, lo que concreta la formación interprofesional.

Palabras clave: Salud, Pasantía no médica, Especialización, Educacion universitaria, Capacitación profesional.

\section{INTRODUÇÃO}

O trajeto de construção do Sistema Único de Saúde (SUS), no decorrer dos anos, constata a ampliação do conceito de saúde, além da expansão de suas ações com o propósito de abranger intervenções capazes de garantir a integralidade da assistência à saúde. Verificou-se que para obter essa garantia seria necessário incluir os diferentes tipos de serviços e níveis de atenção à saúde aos mais variados profissionais, numa perspectiva de interdisciplinaridade; que articula os diferentes saberes e práticas com o objetivo de produzir um bem comum, sem desvalorizar o saber e a atribuição de cada profissão (CEZAR PK, et al., 2015).

Os serviços públicos de saúde no Brasil têm importante papel para a formação de profissionais, isso ganha relevância com a instituição do SUS, que integra práticas de pesquisa e ensino em saúde para estabelecer seu modelo de assistência constituído nos princípios e diretrizes que organizam o sistema (CEZAR PK, et al., 2015).

A residência em saúde é um curso de Pós-Graduação lato sensu, que surgiu nos Estados Unidos da América, no ano de 1879 e no Brasil no ano de 1945, na Universidade de São Paulo (USP), ambas ofertadas para o curso de Medicina (FARJADO AP, et al., 2010). Sua regulamentação ocorreu por meio do decreto presidencial no 80.281, com a criação da Comissão Nacional de Residência Médica (CNRM), no dia cinco de setembro de 1977 (BRASIL, 1977).

As Residências Multiprofissionais em Saúde (RMS) foram instituídas no Brasil, em 2005, a partir da promulgação da Lei $n^{\circ} 11.129$, com a finalidade de formar coletivamente equipes em serviço, contribuindo para a integralidade no atendimento ao usuário, contemplando todos os níveis de atenção à saúde e gestão do sistema em articulação com as outras modalidades de residências: uniprofissional (direcionadas apenas para uma categoria profissional) e médica (BRASIL, 2005). As características e objetivos estão definidos de forma sucinta na legislação de oㅜ 11.129 de 30 de Junho de 2005 conforme o Art. 13:

Art. 13. Fica instituída a Residência em Área Profissional da Saúde, definida como modalidade de ensino de pós-graduação lato sensu, voltada para a educação em serviço e destinada às categorias profissionais que integram a área de saúde, excetuada a médica.

$\S 10$ A Residência a que se refere o caput deste artigo constitui-se em um programa de cooperação intersetorial para favorecer a inserção qualificada dos jovens profissionais da saúde no mercado de trabalho, particularmente em áreas prioritárias do Sistema Único de Saúde. 
$\S 20$ A Residência a que se refere o caput deste artigo será desenvolvida em regime de dedicação exclusiva e realizada sob supervisão docente assistencial, de responsabilidade conjunta dos setores da educação e da saúde (BRASIL, 2005).

Com a criação da Comissão Nacional de Residência Multiprofissional em Saúde (CNRMS) pela Portaria Interministerial, Ministério da Educação (MEC) e Ministério da Saúde (MS), no 45/2007 ficam definidos os princípios e diretrizes da RMS. A CNRMS tem a função de coordenação das ações e habilitação dos Programas; o Ministério da Educação (MEC) é responsável por fornecer suporte técnico e administrativo e, em parceria com o Ministério da Saúde (MS) participa do financiamento, estrutura e funcionamento da CNRMS (BRASIL, 2007).

Dados do Sistema da Comissão Nacional de Residência Multiprofissional em Saúde (SisCNRMS) indicam que 212 programas de residência multiprofissionais estavam cadastrados no ano de 2012. Neste mesmo ano, quando comparado ao anterior, houve crescimento de $188 \%$ de residências em Hospitais Universitários Federais (BRASIL, 2012).

Com a autorização de 7.425 bolsas no ano de 2011 e 6.038 em 2012 o MEC refere ter investido, $R \$ 235,5$ milhões no pagamento de bolsas para programas de residência só no ano de 2011 , entre residências médicas, uniprofissionais e multiprofissionais, sendo a modalidade multiprofissional equivalente a $16 \%$ do total de bolsas concedidas (BRASIL, 2012), abrangendo as seguintes profissões: Biomedicina, Ciências Biológicas, Educação Física, Enfermagem, Farmácia, Fisioterapia, Fonoaudiologia, Medicina Veterinária, Nutrição, Odontologia, Psicologia, Serviço Social e Terapia Ocupacional.

Os Programas têm como objetivo formar profissionais para uma atuação diferenciada no SUS, uma vez que pressupõe construção interdisciplinar dos profissionais em saúde, trabalho em equipe, dispositivo de educação permanente e reorientação das lógicas tecnoassistenciais (BRASIL, 2005) e (BRASIL, 2004). Difundir a formação em serviço foi fundamental para a expansão dos programas de residência multiprofissional em saúde (PRMS), que passam a ocupar as instituições e serviços como dispositivo de constituição de práticas baseadas na integralidade (CEZAR PK, et al., 2015).

A duração da RMS é de dois anos, sendo estes de dedicação exclusiva (DE), com carga horária total de 5.760 horas, $80 \%$ dessa carga horária total deve ser efetuada em atividades práticas e teórico-práticas, com ações de integração, educação, gestão, atenção e participação social, e os $20 \%$ restantes são destinados à atividade teórica, perfazendo uma carga horária de 60 horas semanais (BRASIL, 2014).

Considerando que a proposta de formação na modalidade de residência multiprofissional está crescendo e ganhando mais espaço dentro dos serviços de saúde, faz-se imprescindível investir em pesquisas que caracterizem os programas e sua abrangência no ensino em saúde, reveladas como iniciativas que desenvolvam a reorientação das práticas entre ensino, serviço e comunidade, voltadas à humanização em saúde.

Diante disso, esta pesquisa tem como questão norteadora: Como se caracterizam as residências multiprofissionais em saúde do Brasil? O objetivo deste estudo foi de caracterizar os programas de residências multiprofissionais em saúde do Brasil.

\section{MÉTODOS}

Trata-se de um estudo quantitativo, descritivo, transversal, exploratório. Os dados foram coletados durante o período de março a setembro de 2019. A pesquisa foi realizada a partir de um banco de dados cedido pelo Ministério da Educação (MEC), bem como pela busca na internet, através dos editais e sites dos programas identificados.

Foram incluídos na amostra todos os programas de residência multiprofissionais no Brasil, ativos, cadastrados no MEC e acessíveis através da internet, sendo excluídos os Programas de Residências que se encontravam inativos. As variáveis analisadas foram: região geográfica, estado brasileiro, financiamento, quantitativo de vagas, ano de início do programa, área de concentração, instituição propositora, profissões inseridas nos programas e cenários de práticas. 
Todos os dados coletados foram transcritos, armazenados em planilhas eletrônicas do programa Microsolft Excel 2010 e analisados descritivamente. Os resultados foram apresentados em formato de gráficos e tabelas e discutidos com a literatura atual disponível sobre o tema. Os dados utilizados neste estudo são de domínio público secundários e não envolvem contato direto com seres humanos e animais, bem como não identificam os participantes. Diante disso, não foi necessário ser submetido à aprovação em Comitê de Ética em Pesquisa, ademais, os dados consultados estão disponíveis na internet, para todas as pessoas que se dispuserem a procurá-los.

\section{RESULTADOS}

Foram encontrados 416 programas de residências multiprofissionais, e sua distribuição por região, onde a maioria está localizado nas regiões Sudeste (34\%) e Nordeste (26\%) (Figura 1).

Figura 1 - Distribuição dos programas de residência por região do país em $(\%)(n=416)$.

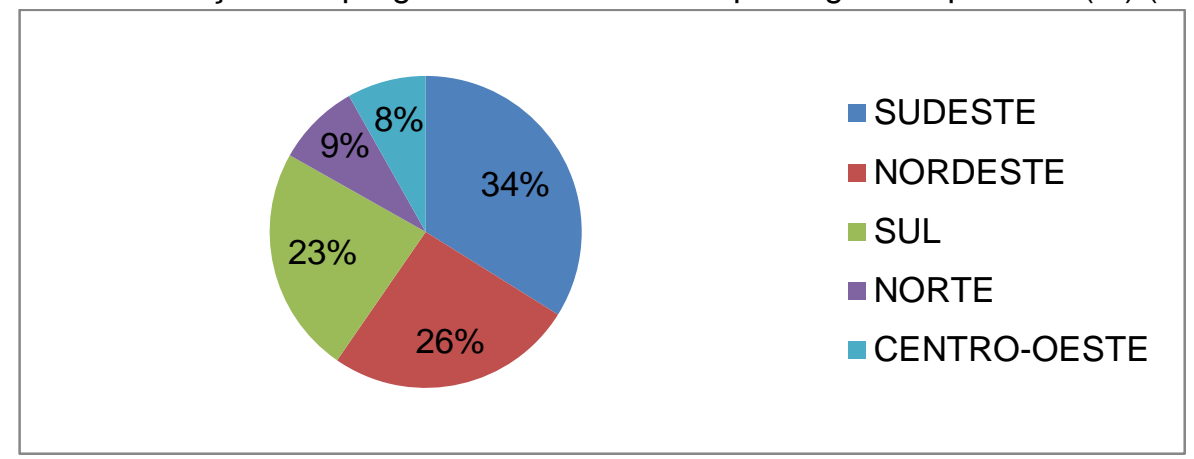

Fonte: Silva MB, et al., 2020.

Na distribuição dos programas de residência por Estado brasileiro, o Estado de São Paulo (SP) aparece com $20 \%$ da totalidade dos programas. Alagoas (AL) e Acre (AC) com 0,5\%, Roraima (RR) e Amapá (AP) não apresentaram porcentagem (Figura 2).

Figura 2 - Distribuição dos programas de residência por Estado em $(\%)(n=416)$.

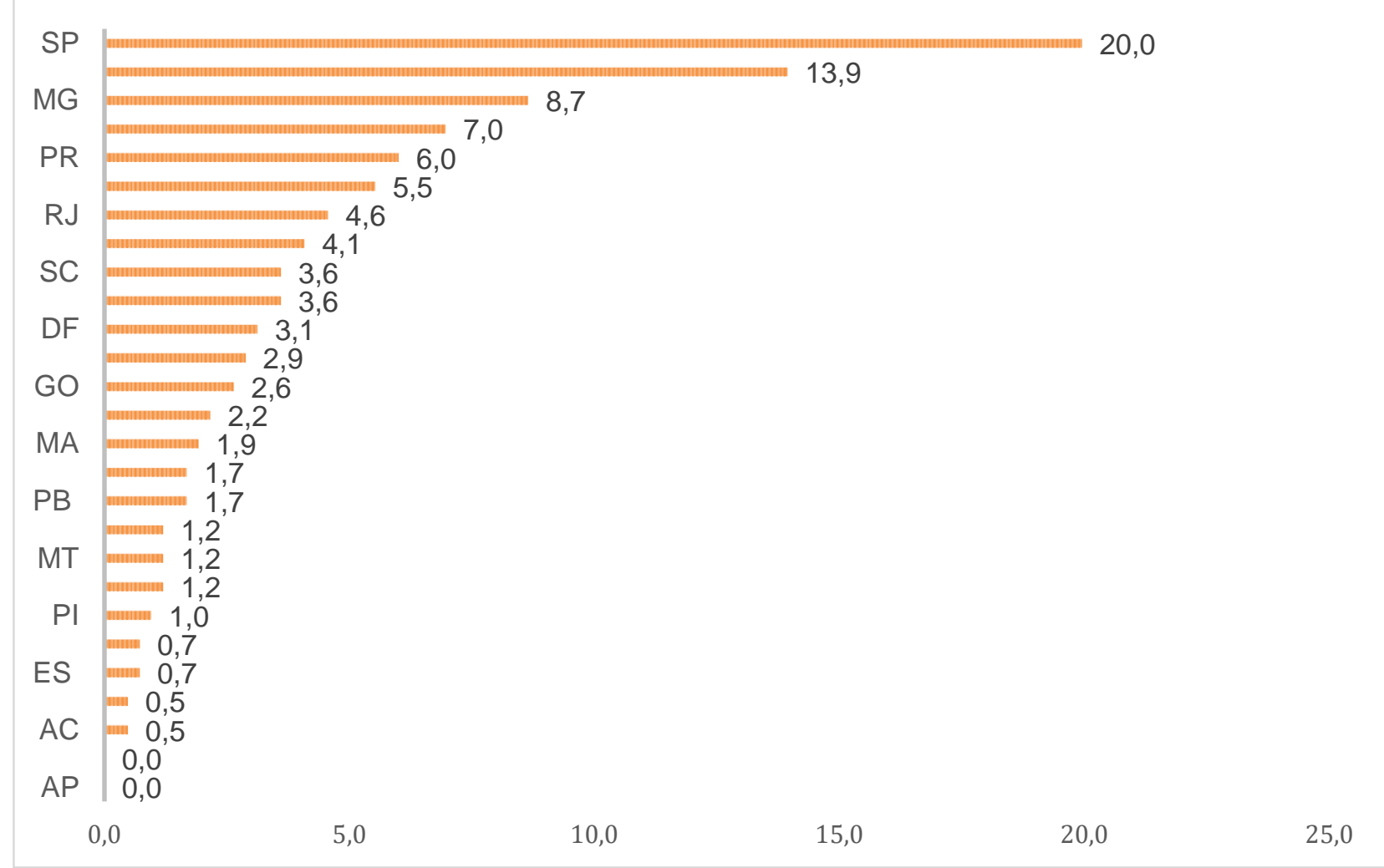

Fonte: Silva MB, et al., 2020. 
Os dados na tabela 1 evidenciam que $60,0 \%$ dos programas de residência encontrados são financiados pelo MS, seguido de $33,7 \%$ do financiamento pelo MEC. Porém, um expressivo percentual de $38,70 \%$ dos editais não constatava o tipo de financiamento em seus editais (Tabela 1).

Em relação ao quantitativo de vagas ofertadas por programa, $59,4 \%$ ofertam 10 ou mais vagas. Destacase, com $85,58 \%$, a ausência de informações sobre o ano de início dos programas de residências. Em relação ao tipo de instituição $77,88 \%$ provém do serviço público e $6,01 \%$ de instituições sem fins lucrativos.

Tabela 1 - Distribuição dos programas de residência multiprofissionais quanto ao financiamento, quantidade de vagas, ano de início do programa e instituição propositora $(n=416)$.

Variável

Categorias
$(\%)$

Ministério da saúde (MS) $\quad 60,0 \%$

$\begin{array}{lll}\text { Financiamento } & \text { Ministério da educação (MEC) } \quad 33,7 \%\end{array}$

Outros tipos de financiamento $\quad 6,3 \%$

De 1 à 5 vagas

$10,7 \%$

Quantidade de vagas

De 6 à 9 vagas

$29,9 \%$

De 10 ou mais vagas

$59,4 \%$

2005 à 2009

$43,3 \%$

Ano de início do

2010 à 2014

$36,7 \%$

programa

2015 à 2018

$20,0 \%$

Pública

$77,88 \%$

Instituição propositora

Privada

$16,11 \%$

Sem fins lucrativos

$6,01 \%$

Fonte: Silva MB, et al., 2020.

Em relação ao eixo de concentração, verificou-se que $22,8 \%$ dos programas de residência, pertenciam ao eixo da Saúde da Família/Atenção Básica/Saúde Coletiva. Destaca-se ainda que as áreas de concentração cuidados paliativos e transplante de órgãos já aparecem, apesar do pequeno percentual de $0,7 \%$ cada (Figura 3). 
Figura 3 - Distribuição dos programas de residência por área de concentração em (\%). (n= 416).

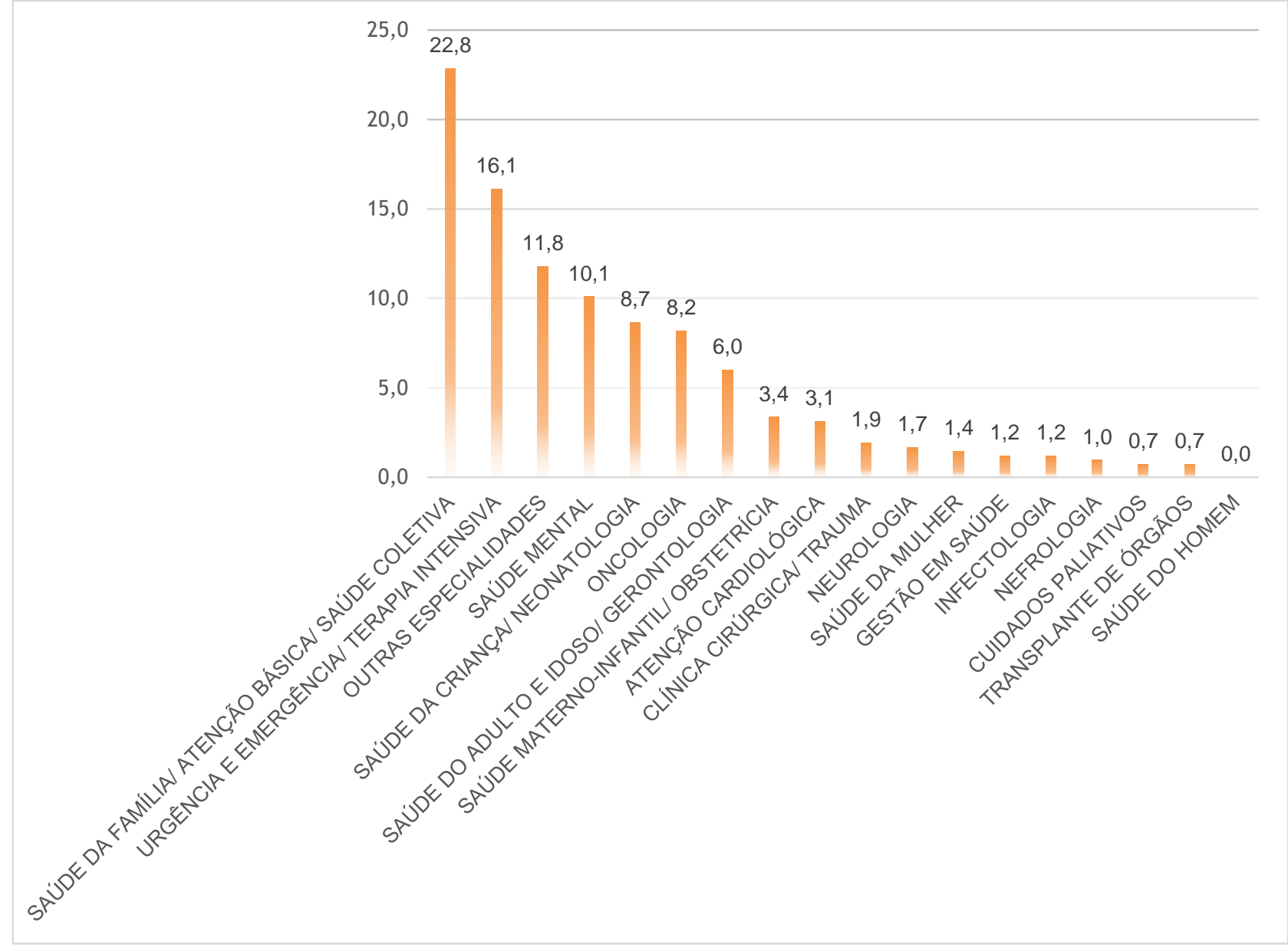

Fonte: Silva MB, et al., 2020.

A categoria profissional da Enfermagem se destaca estando presente em 93,8\% dos programas de residência, já a Biomedicina aparece com 5,8\%, obtendo assim a menor porcentagem. No entanto, observouse que todas as profissões de saúde já aparecem inseridas dentro dos programas, de modo geral (Figura 4).

Figura 4 - Distribuição das profissões nos programas de residência em (\%). (n= 416).

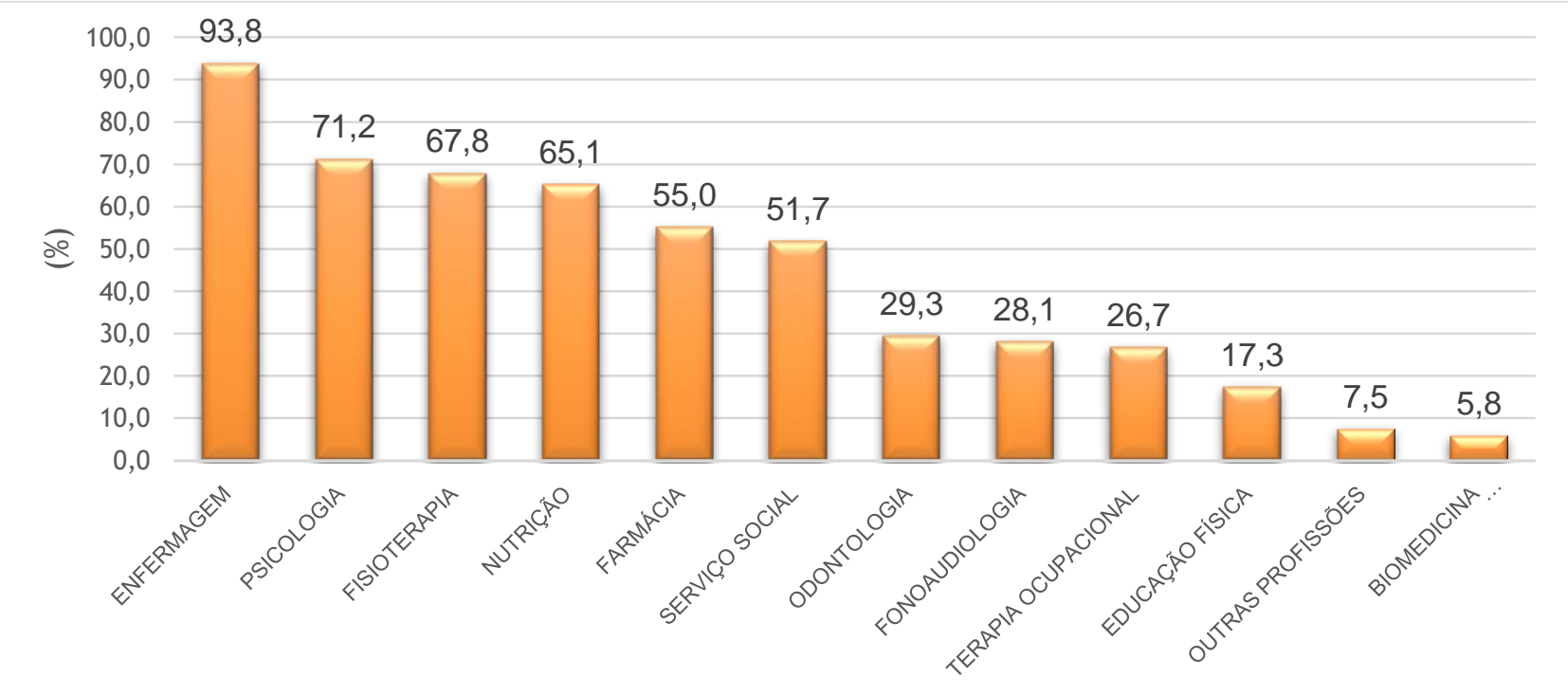

Fonte: Silva MB, et al., 2020. 
Considerando os cenários de prática, $88,9 \%$ dessa informação estava ausente nos editais, mas, nos que estavam disponíveis, foi encontrado que $1,8 \%$ dos residentes atuavam nos centros cirúrgicos e $30,1 \%$ em Unidades Básicas de Saúde (Figura 5).

Figura 5 - Distribuição dos cenários de prática nos programas de residência em (\%). ( $n=416)$.

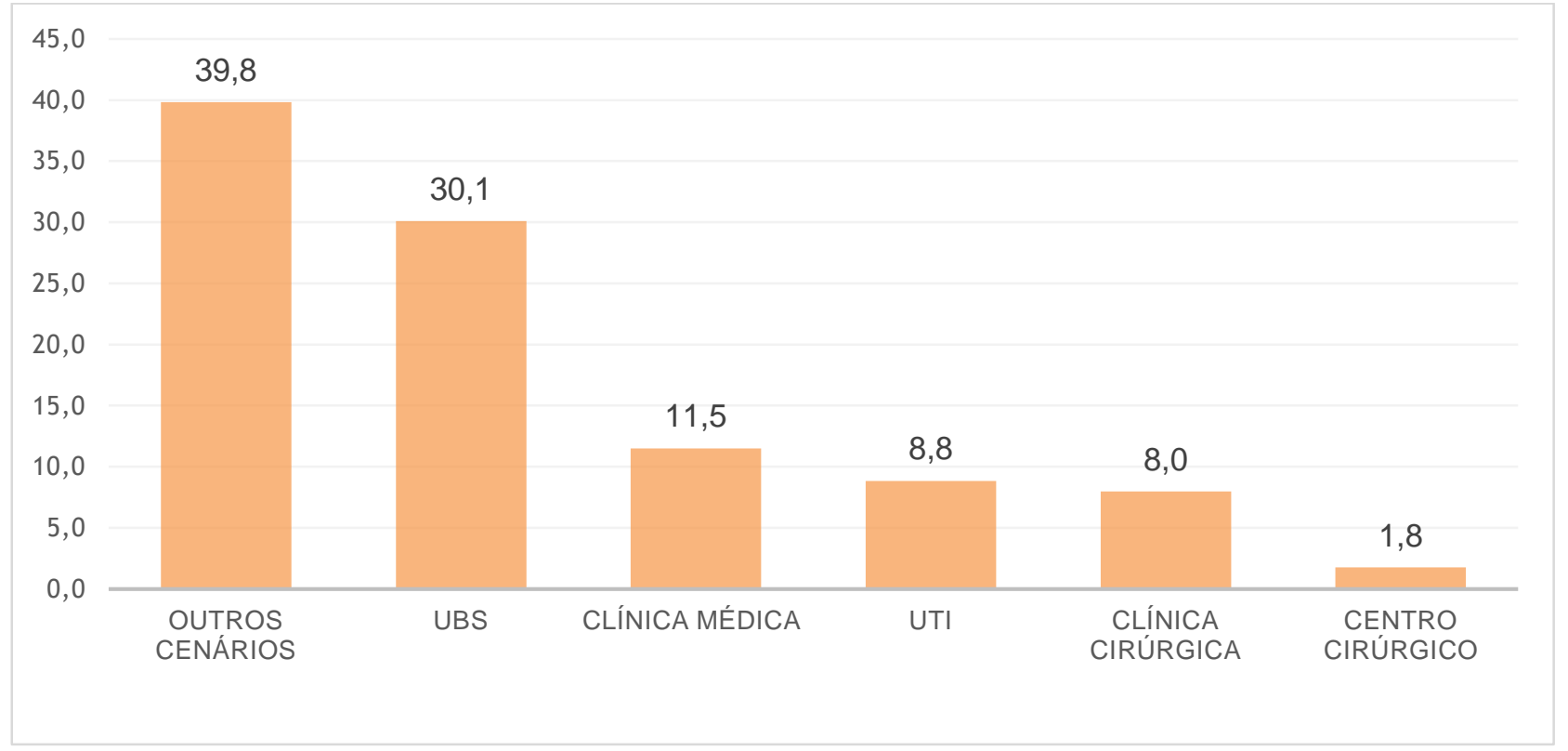

Fonte: Silva MB, et al., 2020.

\section{DISCUSSÃO}

A partir da análise dos dados levantados, evidenciou-se que $34 \%$ dos programas de residência identificados estão localizados na região Sudeste, $26 \%$ na região Nordeste e $23 \%$ na região Sul; já a região Norte obteve apenas $8 \%$ dos programas. Resultado similar foi encontrado em uma coletânea realizada em 2006 pelo MS por intermédio do Departamento de Gestão da Educação na Saúde (DEGES) acerca das residências multiprofissionais em saúde, onde aparece 206 vagas para a região Sudeste (em maior concentração), região Sul com 171 vagas, região Nordeste com 125 vagas e, por último, a região Norte com apenas 14 vagas (BRASIL, 2006).

Acrescenta-se, ainda, em relação à distribuição dos programas, agora a partir dos Estados, uma maior centralização dos PRMS nos Estados de São Paulo (20\%) e do Rio Grande do Sul (13,9\%). Observou-se, em um estudo publicado no ano de 2017, concordância com os dados apresentados, onde a região Sudeste foi a mais contemplada com os programas. Isso espelha o alto desempenho econômico dessa região, que se configura como o centro dinâmico do mercado de trabalho de saúde no País (VIERIA ALS, et al., 2004).

Durante o primeiro lançamento dos editais de convocação, em 2009, o MS estabeleceu três regiões como prioritárias para o financiamento dos programas: Norte, Nordeste e Centro-Oeste. Entretanto, a única região que apresentou um significativo número de programas foi o Nordeste, só ficando atrás do Sudeste que representou 20,6\% dos programas validados. Nos anos subsequentes, 2010, 2011 e 2013 a segunda região com mais PRMS em atividade foi o Nordeste que, só foi superado no ano de 2014, pela região Sul que passou a ter $25,5 \%$ dos programas aprovados (SARMENTO LF, et al., 2017).

Essas representações convergem com a situação da educação superior no Brasil, que se retém, historicamente, nos grandes centros urbanos, principalmente no eixo Sul-Sudeste. Dados das matrículas do ano de 2013, nos cursos do ensino superior presenciais, tanto na rede pública quanto privada, mostram que as regiões Sudeste, Nordeste e Sul matricularam $47,2 \%, 20,9 \%$ e $15,6 \%$, respectivamente, enquanto as regiões Centro-Oeste e Norte, de modo respectivo, foram responsáveis por apenas $9,4 \%$ e 6,9\% do total de matriculados durante esse período (BRASIL, 2014). 
Tal configuração demanda maior controle entre as instituições de ensino superior e os sistemas de saúde que, além de formar profissionais com qualificação e em quantidade eficaz para suprir as necessidades da população, precisam reparar as desigualdades e as diversidades existentes dos indicadores de saúde, com o objetivo de superá-las (DAL POZ MR, et al., 2015).

Segundo este estudo, no que se refere ao financiamento, $(60,0 \%)$ dos recursos provém do MS, $(33,7 \%)$ do MEC, enquanto apenas (6,3\%) provém de outras fontes. De acordo com o MS, investir nas RMS tem como objetivo, em suas potencialidades de formação política e pedagógica, subsidiar a construção dos profissionais, além de contribuir com a transformação do desenho técnico-assistencial do SUS, onde a residência se torna um espaço privilegiado de mudança por se tratar de um processo de formação no e pelo trabalho (SILVA LC, 2017).

Silva LB (2018) refere que, o MEC passou a participar do financiamento dos programas de residências multiprofissionais em saúde a partir de 2009, com o lançamento do projeto "Implantação do Programa de Residência Integrada Multiprofissional em Saúde da rede de Hospitais Universitários Federais". Anteposto a esse período, até 2009, o financiamento das residências médica, uni e multiprofissional, era mantido pelo MS. Com isso, analisou-se o crescimento de 700\% das bolsas entre os anos de 2010 e 201412. Esse grande avanço no número de bolsas, aponta a importância da modalidade de formação em serviço, que é proposta pelas residências em saúde, vem tomando ao longo do tempo, com maior relevância desde 2010, após a inserção do MEC nesse processo (SILVA LB, 2018).

Em relação aos eixos de concentração, ocorreu a predominância da Saúde da Família/Atenção Básica/Saúde Coletiva/ com 22,8\% e Urgência e Emergência/Terapia Intensiva 16,1\%. De acordo com o Diário Oficial da União, publicado em 17 de janeiro de 2019, que aborda a "Adesão de Entes Federados e Instituições à Concessão de Bolsas do Ministério da Saúde para Programas de Residência em Área Profissional da Saúde", esses dois eixos estão contidos nas áreas prioritárias para abertura de novos programas. Ressalta ainda, que o MS objetiva financiar campos de atuação de forma estratégica para o SUS, esses, definidos entre os gestores a partir das necessidades identificadas tanto no âmbito local quanto regional (BRASIL, 2019)

$A$ atenção básica $(A B)$ foi efetivada no Brasil como política de Estado, isso ocorreu no decurso da implementação do SUS, onde as ações de atenção primária a saúde (APS) passaram a ser denominadas atenção básica. A partir da organização da rede de forma hierarquizada e em nível de complexidade crescente, ela é tida como a principal porta de entrada do SUS e, compreende a integralidade da saúde, com ações de promoção e proteção da saúde, prevenção de agravos, riscos e doenças, diagnóstico, tratamento e reabilitação da saúde. Conceito este previsto nas normas que dispõe o SUS e na Constituição Federal do Brasil de 1988 (BRASIL, 2017).

A Portaria № 2.436, de 21 de setembro de 2017 aprova a Política Nacional de Atenção Básica, estabelecendo a revisão de diretrizes para a organização da Atenção Básica, no âmbito do Sistema Único de Saúde (SUS). Em seu Artigo 3ํㅡ traz os princípios e Diretrizes do Sus e das Redes de Atenção à Saúde (RAS) para operacionalizar na $\mathrm{AB}$ :

Art. $3^{\circ}$ São Princípios e Diretrizes do SUS e da RAS a serem operacionalizados na Atenção Básica: I - Princípios: a) Universalidade; b) Equidade; e c) Integralidade. II Diretrizes: a) Regionalização e Hierarquização: b) Territorialização; c) População Adscrita; d Cuidado centrado na pessoa; e) Resolutividade; f) Longitudinalidade do cuidado; g) Coordenação do cuidado; h) Ordenação da rede; e i) Participação da comunidade.

A ocorrência do crescimento de violências, acidentes e doenças crônicas, acarreta no aumento de atendimentos nas portas dos hospitais, segundo infere Brasil (2015). E que ao analisar as normas que regulamentam a Política Nacional de Atenção às Urgências, evidencia que a atenção às urgências no Brasil foi centrada na atenção hospitalar, a partir da implantação das Centrais da Regulação Médica de Urgências e do Serviço de Atendimento Móvel de Urgência (SAMU) nos grandes centros urbanos, desde a segunda metade da década de 1990. 
Para o Brasil (2014), com a participação do MEC, além do acréscimo no número de bolsas, houve novo delineamento dos programas de residência, agora voltados para atuação hospitalar. Mendes AG (2010) relata que os recursos das bolsas foram deslocados da atenção básica e da saúde mental para a atenção de média e alta complexidades, o que retrata a oferta dos atendimentos dos hospitais de ensino.

A Política Nacional de Atenção às Urgências (PNAU) foi criada em 2003, com o intuito de diminuir as lacunas assistenciais entre a AB e a média e alta complexidade (BRASIL, 2003). Ademais, também baseada nos princípios da rede, busca suprir outras necessidades, como a implantação dos sistemas de regulação. Com isso, propiciar atendimento integral aos pacientes em todos os níveis de atenção (GRANJA GF, et al., 2013).

As categorias profissionais inseridas no processo dos programas de residência multiprofissionais em saúde ganham evidência, onde grande parte das profissões já se apresentam com porcentagens significativas. Destacando-se a profissão da enfermagem que obteve $93,8 \%$.

Segundo dados do Conselho Federal de Enfermagem, CONFEN (2016) o Brasil tem cerca de 1000 programas de graduação em enfermagem, desses, são formados em torno de 50.000 enfermeiros anualmente, perfazendo um total de mais de 400.000 enfermeiros atualmente. É o país que aparece com o maior número de escolas de enfermagem na Região das Américas e programas de pós-graduação em enfermagem. Para Nascimento ZP (1976), o profissional enfermeiro faz parte da composição da equipe multiprofissional no sistema de saúde e, ajuda a planejar e executar os programas e políticas. É um importante membro para identificar as demandas do paciente, a partir de sua percepção e aproximação com o indivíduo e coletividade na promoção da saúde.

Paiva SP, et al. (2019) explana que além de ser um espaço privilegiado para formação especializada em saúde, os programas de residências multiprofissionais em saúde propõem a importância do trabalho coletivo, implicando assim, na formação de saberes, perspectivas de análise e trabalho interdisciplinar. A individualidade do trabalho consiste na diminuição da eficiência das ações, atingindo em consequência os pacientes. Para Costa MJC (1978), quando as atividades são integradas, as realizações se multiplicam e os perigos são reduzidos. Ou seja, o desenvolvimento do trabalho em equipe não pode ser apenas o resultado do arranjo de diversos profissionais interdisciplinares, mas precisa obedecer a um padrão com coerência e ter um plano bem estudado entre os envolvidos.

Por outro lado, apesar de estudos encontrados por Paiva SP, et al. (2019) admitirem a notabilidade do trabalho multiprofissional, os mesmos ainda ressaltam que há entraves e desafios para desconstrução da lógica disciplinar e fragmentada que ainda se faz presente na formação e no trabalho em saúde com a forma hegemônica do saber biomédico neste campo.

Ressalta-se a importância dos PRMS, que nascem com capacidade de construção de uma formação em saúde, com o intuito de atender as necessidades complexas postas no cotidiano do SUS. Enquanto proposta de formação, tem em sua esfera a construção de práticas integradas entre as profissões com habilidade para enfrentar a fragmentação dos saberes e fazeres ainda caracterizados nas políticas e no trabalho em saúde (SILVA LB, 2018).

\section{CONCLUSÃO}

As residências multiprofissionais em saúde no Brasil concentram-se na região sudeste, principalmente São Paulo, são financiadas em sua maioria pelo MS, ofertam prioritariamente 10 vagas ou mais; as profissões mais constantes são enfermagem e psicologia e a atenção básica é a área de concentração mais comum. Esses resultados mostram que essa modalidade de pós-graduação existe em todo o país e oportuniza um conhecimento essencial para o desenvolvimento da interprofissionalidade aos profissionais da área da saúde. Por se tratar de algo relativamente novo enquanto formação profissional, as produções científicas para embasamento das discussões sobre essa modalidade de pós-graduação lato sensu são principiantes. Isso posto, destaca-se a importância para realização de mais estudos que corroborem para o destaque dessa modalidade de formação. 


\section{REFERÊNCIAS}

1. BRASIL. Conselho Nacional de Secretários de Saúde. Rede de Atenção às Urgências e Emergências: Avaliação da Implantação e do Desempenho das Unidades de Pronto Atendimento (UPAs). Brasília: CONASS, 2015. 400 p.

2. BRASIL. Decreto no 80.281 , de 5 de setembro de 1977. Regulamenta a Residência Médica, cria a Comissão Nacional de Residência Médica e dá outras providências. Diário Oficial da União, 6 de set. 1977.

3. BRASIL. Lei no 11.129, de 30 de junho de 2005. Institui o Programa Nacional de Inclusão de Jovens - Projovem; cria o Conselho Nacional de Juventude (CNJ) e a Secretaria Nacional da Juventude; altera as Leis no 10.683, de 28 de maio de 2003, e 10.429, de 24 de abril de 2002; e dá outras providências. Diário Oficial da União, 1 de jul. 2005.

4. BRASIL. Ministério da Educação (BR). A democratização e expansão da educação superior no país $2003-2014$. Brasília: MEC; 2014.

5. BRASIL. Ministério da Educação (BR). Secretaria de Educação Superior. Prestação de Contas Ordinárias Anual. Relatório de Gestão do Exercício de 2011. Brasília: MEC; 2012.

6. BRASIL. Ministério da Saúde. Adesão de entes federados e instituições à concessão de bolsas do Ministério da Saúde para Programas de Residência em área profissional da saúde. Diário Oficial da República Federativa do Brasil. Ministério da Saúde/Secretaria de Gestão do Trabalho e da Educação na Saúde; 2019.

7. BRASIL. Ministério da Saúde. Política de educação e desenvolvimento para o SUS: caminhos para a educação permanente e pólos de educação permanente em saúde. Brasília: MS; 2004.

8. BRASIL. Ministério da Saúde. Portaria de Consolidação no 02, Anexo XXII, de 28 de setembro de 2017. Aprova a Política Nacional de Atenção Básica. Diário Oficial da República Federativa do Brasil. 2017.

9. BRASIL. Ministério da Saúde. Portaria no 1.863, de 29 de setembro de 2003. Institui a Política Nacional de Atenção às Urgências e dá outras providências. Diário Oficial da República Federativa do Brasil. 2003.

10. BRASIL. Ministério da Educação, Ministério da Saúde (BR). Portaria Interministerial no 45, de 12 de janeiro de 2007. Dispõe sobre a Residência Multiprofissional em Saúde e a Residência em Área Profissional da Saúde e institui a Comissão Nacional de Residência Multiprofissional em Saúde. Diário Oficial da União, Brasília: MS; 2007.

11. BRASIL. Ministério da Saúde. Secretaria de Gestão do Trabalho e da Educação na Saúde. Departamento de Gestão da Educação na Saúde. Residência multiprofissional em saúde: experiências, avanços e desafios. Brasília: MS; 2006.

12. BRASIL. Secretaria de Educação Superior. Comissão Nacional de Residência Multiprofissional em Saúde. Resolução CNRMS nำ 5, de 7 de novembro de 2014. Dispõe sobre a duração e a carga horária dos programas de Residência em Área Profissional da Saúde nas modalidades multiprofissional e uniprofissional e sobre a avaliação e a frequência dos profissionais da saúde residentes. Diário Oficial da República Federativa do Brasil; 2014.

13. CEZAR PK, et al. A psicologia na estratégia de Saúde da Família: vivências da residência multiprofissional. Psicol Ciênc Prof, 2015; 35(1): 211-224.

14. CONSELHO FEDERAL DE ENFERMAGEM (COFEN). Enfermagem em Foco 2016; (7).

15. COSTA MJC. Atuação do Enfermeiro na Equipe Multiprofissional. Rev. Bras. Enferm, 1978; 31(3).

16. DAL POZ MR, et al. Formação em saúde: problemas e tendências. Fundação Oswaldo Cruz, Rio de Janeiro. 2015. $10 \mathrm{p}$.

17. FARJADO AP, et al. Brasil. Ministério da Saúde. Grupo Hospitalar Conceição Residências em saúde: fazeres \& saberes na formação em saúde. Porto Alegre: Hospital Nossa Senhora da Conceição, 2010; 260p.

18. GRANJA GF, et al. Análise da Política Nacional de Atenção às Urgências no SUS: avanços e desafios na efetivação das redes de atenção à saúde. In: Congresso Brasileiro de Política, Planejamento e Gestão em Saúde. Abrasco, SP. 2013; 2: 116p.

19. LOBATO CP. Formação dos trabalhadores de saúde na residência multiprofissional em saúde da família: uma cartografia da dimensão política (Dissertação). Londrina (PR): Universidade Estadual de Londrina. Programa de PósGraduação em Saúde Coletiva; 2010.

20. MENDES AG. Serviço Social, saúde e interdisciplinaridade. In: Silva, LB; Ramos, A. Serviço Social, saúde e questões contemporâneas: reflexões críticas sobre a prática profissional. São Paulo: Papel Social, 2013:64-49. Sodré, F. Serviço Social e o campo da saúde: para além de plantões e encaminhamentos. Serviço Social e Sociedade, São Paulo, 2010; 103: 475-453.

21. NASCIMENTO ZP. O Enfermeiro- Membro da Equipe Multiprofissional. Rev. Paulista de Hospitais, São Paulo, 1976; 24(5): 213-15.

22. PAIVA SP, et al. O serviço social e o trabalho em equipe multiprofissional nas residências em saúde: estado da arte. Revista Libertas, Juiz de Fora, 2019; (2): 497-482.

23. SARMENTO LF, et al. A distribuição regional da oferta de formação na modalidade Residência Multiprofissional em Saúde. Saúde Debate, Rio de Janeiro, 2017; 41(113): 424-415.

24. SILVA LB. Residência Multiprofissional em Saúde no Brasil: alguns aspectos da trajetória histórica. R. Katál. Florianópolis, 2018; 21(1): 209-200.

25. 25. SILVA LC. Residência multiprofissional no contexto do Sistema Único de Saúde: perspectivas e contradições. Revista Libertas, Juiz de Fora, 2017; 17(1): 109-132.

26. VIERIA ALS, et al. Mercado de trabalho em saúde na região sudeste-brasil: a inserção da equipe de enfermagem. Rev Latino-americana de Enfermagem, Ribeirão Preto, 2004; 12(1): 138-134. 